Proceedings of the 2006 Winter Simulation Conference

L. F. Perrone, F. P. Wieland, J. Liu, B. G. Lawson, D. M. Nicol, and R. M. Fujimoto, eds.

\title{
OPTIMAL CONTROL OF MAKE-TO-ORDER MANUFACTURING SYSTEMS VIA SELECTED ORDER ACCEPTANCE
}

\author{
Amitava Nandi \\ SR \& ED Division \\ Canada Revenue Agency \\ 333 Laurier Avenue West \\ Ottawa, Ontario, K1A 0L9, CANADA
}

\author{
Paul Rogers \\ Dept. of Mechanical \& Manufacturing Engineering \\ 2500 University Drive N.W. \\ University of Calgary \\ Calgary, Alberta, T2N 1N4, CANADA
}

\begin{abstract}
Capacity constrained make-to-order manufacturing systems with exogenously set due dates and heavy tardiness penalties can be effectively managed by selective acceptance of orders, especially when the system encounters heavy congestion. This is demonstrated using a popular order acceptance rule. How this rule can optimally control a manufacturing system under different environments and how the main performance measures of the manufacturing system are affected in doing so, are demonstrated and analyzed. The study is done on a simulated hypothetical manufacturing system used as a testbed.
\end{abstract}

\section{INTRODUCTION}

Order Review and Release (ORR) in manufacturing has long passed its infancy and is now a widely accepted research topic. The rich repository of research in ORR is evident from the expanding portfolio of publications since the days of Wight (1970) when the author proposed the rudimentary principle in production control i.e. not to input jobs into a manufacturing system at a higher rate than the system output. Since then, various ORR strategies have been experimented with in numerous experimental scenarios. Bergamaschi et al. (1997) is one survey paper classifying this volume of research.

In capacity-constrained environments with exogenous due dates and heavy tardiness penalties, the extreme input control approach is a necessity, especially at the time of demand surge. Extreme input control approaches have been studied by queuing theorists as well as applied simulationists. Some early work in queuing theoretic models permitting limited rejection of a portion of demand were done by Lippman (1975), Lippman and Ross (1971), Miller (1969), Scott (1969), and Scott (1970). Relatively recently, on the experimental side, several researchers have conducted simulation experiments of facilities with the capability to utilize this form of extreme control. Philipoom and Fry (1992) studied two order acceptance strategies and found selective acceptance of orders can yield dramatic improvement in the case of capacity-constrained make-toorder (MTO) manufacturing systems. ten Kate (1992) also found that in severe conditions with short lead times, high utilization rate, order acceptance integrated with scheduling function performs better that the situation when they are not integrated. Wester et al. (1994) compared three basic order acceptance approaches and concluded that under heavy workload the one based on detailed scheduling of accepted orders outperforms other aggregate or myopic approaches. Ivanescu et al. (2002) suggested that the choice of the best order acceptance policy should depend on the processing time variability. Nandi and Rogers (2003) tested a two-stage input control mechanism in a capacity-constrained MTO setting with fixed customer due dates where selectively accepted orders can be delayed in a pre-shop pool and opportunistically released to the shop floor. Holding orders in the pre-shop pool was not found to improve the due date performance. Ebben et al. (2005) tested an "aggregate" approach versus an approach developing a complete schedule after each job was accepted. The authors suggested that the aggregate approaches do not work all that well in cases of high demand and little slack (i.e. high due date tightness). Additionally, Nandi and Rogers (2004) developed a simulation-based order acceptance approach, which inherently utilizes the full information of the manufacturing system and demonstrated the concept of optimal control of the manufacturing system under different environments.

This paper is in line with the research activities in the field of extreme input control. Nandi and Rogers (2004) has already demonstrated the concept of optimal control of MTO manufacturing systems using an extreme input control approach, when the system is functioning in an uncontrolled environment. In this paper, this investigation has been further extended to another popular order acceptance rule (described in subsection 2.2), which was earlier investigated by Philipoom and Fry (1992) to some extent, but 


\section{Nandi and Rogers}

was not really studied for optimal control of an MTO manufacturing system. The motivation of this paper lies in this very fact.

The remainder of the paper is structured as follows. First, the order acceptance rule is described, together with a description of the simulated manufacturing environment in which it was tested. A preliminary study on how the control parameters of the order acceptance rule influence the main performance measures of the system is done next. This is followed by an analysis of the optimal control policy of the manufacturing system using this rule under varied environmental conditions. This study is also extended to demonstrate the effect of optimal control on the main performance measures of the system. Finally, some conclusions and possible directions for future work are highlighted.

\section{ORDER ACCEPTANCE RULE}

In this section we first describe the hypothetical manufacturing system within which the order acceptance rule has been implemented. The order acceptance rule used in this study is described next in detail.

\subsection{Description of the System}

The manufacturing system considered here, is identical to the one used in Nandi and Rogers (2003). Please refer to this paper for more details on the system. The manufacturing facility is a make-to-order manufacturing system comprising of 10 machines arranged in 4 workstations with the even numbered workstations having 3 machines and the odd numbered workstations having 2 machines. The total number of operations will vary between 1 and 4 . Each of the 10 machines has a known mean processing time, with actual times being sampled from a Gamma distribution. The jobs in a machine queue are prioritized according to the well-known least-slack-per-remaining-operation dispatching rule, any tie being broken on the basis of first-insystem-first-served. Two classes (viz. regular and urgent) of customer orders are generated with a batch size of unity and inter-arrival times following a Gamma distribution. Orders are selectively accepted following the order acceptance rule in Section 2.2. Each order is assigned an exogenous due date which is a constant flow allowance away from the entry time. This flow allowance is RegFTA or UrgFTA depending on if the order is regular or urgent, RegFTA being greater than UrgFTA. Each accepted order, upon completion, contributes to the system an amount equal to its revenue (Rev) less any incurred tardiness penalty costs $(T C)$. Following are the formulations of Rev.

$$
\operatorname{Rev}=K r \times T W K \text {; for a regular order }
$$$$
\operatorname{Rev}=K u \times T W K \text {; for an urgent order }
$$

Where,

$K r=$ A positive constant

$K u=$ A constant such that $K u>K r$

$T W K=$ Total estimated work content of the order.

Also, following are the formulations of $T C$.

$T C=K t r \times R e v \times$ Tardiness; for a regular order

$T C=K t u \times R e v \times$ Tardiness; for an urgent order

Where,
Ktr
$=$ Tardiness cost factor, a positive con- stant
Ktu $=$ Tardiness cost factor, defined as $K t r \times(\operatorname{RegFTA} / \operatorname{UrgFTA})$
Rev $\quad=\quad$ Net revenue for the order in question (before considering tardiness cost)
Tardiness $=$ Amount of tardiness on completion (which is zero or a positive quan- tity).

So, any rejected job makes no contribution to net revenue but at the same time the opportunity cost of not accepting the job is limited to its revenue. Given the above, the key performance measure of interest for this system, referred to as Overall Percent Achievement (and denoted $O P A)$ is defined as the ratio of actual net revenue to the maximum possible revenue if all jobs were accepted and completed on time. OPA will be less than $100 \%$ due to two sources, cumulative rejection losses, and cumulative tardiness losses. Additionally, two types of loss are tracked individually and referred to by the measures Overall Percent Rejection Loss (denoted OPRL being the ratio of actual cumulative rejection losses to maximum possible revenue), and Overall Percent Tardiness Loss (denoted OPTL being the ratio of actual cumulative tardiness losses to maximum possible revenue). Obviously, $O P A+O P R L+O P T L=$ $100 \%$.

These performance measures are also monitored at the level of order classes. $O P A$ is broken down into UPA and $R P A$. UPA is defined as the ratio of actual net revenue earned by "urgent" orders to maximum possible revenue that could have been earned by "urgent" orders. $R P A$ is the "regular" counterpart of UPA. Similarly, two components of OPRL are UPRL and RPRL. UPRL is the ratio of the loss through rejecting "urgent" orders to the maximum possible revenue that could have been earned by "urgent" orders. $R P R L$ is the "regular" counterpart for UPRL. Last, $U P T L$ and RPTL are the components of OPTL. UPTL is defined as the ratio of the loss through "urgent" tardy orders to the maximum possible revenue that could have been earned by "urgent" orders. Again, RPTL is the "regular" counterpart for UPTL. So, by definition, $U P A+U P R L$ $+U P T L=100 \%$, and also, $R P A+R P R L+R P T L=100 \%$. 
This system can operate under various environmental conditions, which are set by choosing the levels of a number of factors as described in the following subsection 2.1.1.

\subsubsection{Environmental Factors}

(iv) Proportion of urgent orders: This is the proportion of the orders coming into the system that are urgent. This parameter is denoted as PUO.

(v) Due Date Tightness: This is the severity of the pressure of meeting due date requirements in a given situation. In the current research, this is not expressed in a single variable but instead depends upon the values of the flow time allowance (for both "urgent" and "regular" jobs), and the tardiness cost factor. This parameter is denoted as $D D T$.

\subsection{Order Acceptance Rule}

The order acceptance rule that has been used in the work reported in this paper is similar to the path load order review introduced in Philipoom and Fry (1992). This can be stated as follows:

Accept the order if the accepted load on the busiest machine on the candidate order's route is less than a specified maximum value.

For a regular order of type $j$, arriving at time $t$, if $A c$ $c L O M(i)_{t}<R L$, for all $\mathrm{i} \subset q_{j}$, then the order is accepted, otherwise it is rejected; but for an urgent order $R L$ is replaced by $H L$ in this rule. Here,

$$
\begin{aligned}
& \operatorname{AccLOM}(i)_{t}=\quad \begin{array}{l}
\text { The value of the accepted workload } \\
\text { on machine } i \text { at the time of the arri- } \\
\text { val of the order, } t .
\end{array} \\
& q_{j} \quad \begin{array}{l}
\text { The set of all machines on the route } \\
\text { of an order of type } j .
\end{array}
\end{aligned}
$$
$R L$
$=\mathrm{A}$ constant (a control parameter of this rule).
$H L=\mathrm{A}$ constant (another control pa- rameter of this rule).

As noted in Philipoom and Fry (1992), the machine with the heaviest workload would tend to delay the completion of an order more so than less loaded machines. So controlling the input of orders based on this critical machine may make more sense than looking at the total load on the entire shop when making accept/reject decisions. Although this present rule is similar to the one introduced by Philipoom and Fry (1992), this rule further makes a clear distinction in handling urgent and regular orders. As should be obvious from the above description, increasing this rule's control parameters, $R L$ and/or $H L$, should result in less orders being rejected, but may also lead to increased congestion and hence increased order tardiness.

\section{EFFECT OF CONTROL PARAMETERS ON THE MAIN PERFORMANCE MEASURES}

To study the effect of the control parameters on the main performance measures of the system at different values of the environmental factors, $R L$ is varied through $\{14,18$, $22,26,30,34,38\}$ hours with $H L$ fixed at 22 hours. This is done when one of the environmental factors (i.e. $D L, D L V$, $P T V, P U O$ or $D D T$ ) changes across different values while others are held fixed at their base levels. The values of these environmental factors used in this experiment are as follows:

$$
\begin{aligned}
& D L=\{0.75,0.85,0.95\}, \\
& D L V=\{0.1,0.55,1.0\}, \\
& P T V=\{0.1,0.3\}, \\
& P U O=\{0.05,0.15,0.25\}, \\
& D D T=\{\text { Loose", "Tight" } .
\end{aligned}
$$

The base levels of the above five parameters are $\{85 \%, 10 \%, 10 \%, 5 \%$, "Loose" $\}$ in order of their appearance.

A "Loose" level of $D D T$ is characterized by the values of RegFTA $=30$ hours, UrgFTA $=20$ hours, and Ktr $=$ 0.03333 with these values chosen so that they yield $O P A=$ 90\% (approximately) when all orders are accepted and released immediately to the shop floor, with the dispatching rule as first-in-system-first-served under an environment such that all of the environmental factors are at their reference levels. Note that these settings imply that an order will lose all its revenue if it is tardy by its flow allowance.

A "Tight" level of $D D T$ is set with $\operatorname{RegFTA}=21$ hours, UrgFTA $=14$ hours, and Ktr $=0.05952$ so that the system can achieve $O P A=58 \%$ (approximately) when working under the same conditions as in the case of the "Loose" level. Note that with these settings, if a regular or- 
der or an urgent order is tardy by $80 \%$ of its flow time allowance, the order will lose all its revenue.

For each of the different scenarios, the system has been simulated for 5 replications each of length 83520 hours which includes a warm-up period of 11520 hours, so that a confidence interval on the average of each of the observed performance measures has a half width less than or equal to $0.1 \%$ of the mean value of the performance measure. The observed performance measures are $O P A, U P A$, $R P A, O P R L$ and $O P T L$. Figure 1 has been drawn with the environmental factors at their base levels. The figure shows that as $R L$ increases $O P A$ initially increases. The

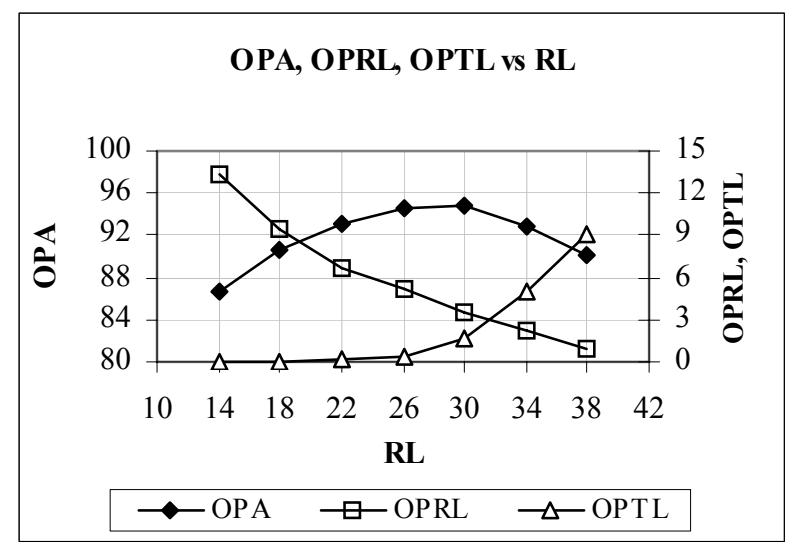

Figure 1: $O P A, O P R L$ and $O P T L$ vs. $R L$

figure also shows how the corresponding $O P R L$ and $O P T L$ vary with increasing $R L$ to yield the resulting $O P A$. As $R L$ increases, $O P T L$ increases while $O P R L$ decreases. If $R L$ increases further, $O P A$ will eventually decline owing to the very high $O P T L$ although $O P R L$ will be very low. So for a fixed value of $H L$, there can be found a $R L$ for which $O P A$ is maximum where the total loss, comprised of rejection loss and tardiness loss, is the minimum for the given set of values of the environmental factors.

To investigate the effect of control parameters on $O P A$, when the environmental factors vary, the above experiment was further extended. In these experiments, one environmental factor is varied while others are kept at their base levels. Also for simplicity $H L$ is kept constant at 22 hours in this experiment. The results are summarized in Table 1.

When $D L$ is varied keeping other environmental factors at their base levels, a convex nature in the values of $O P A$ is observed. As $D L$ increases, the maximum value of $O P A$ is achieved at a lower value of $R L$, which means that at a higher congestion the system will reject more orders to reach the maximum $O P A$. As the present system has a fixed capacity and the due date of the orders cannot be influenced, at a higher $D L$ the system achieves the maximum $O P A$ by rejecting more orders (i.e. by lowering $R L$ ).
As $D L V$ increases, the value of $O P A$ decreases at a particular $R L$. The interaction effect of $R L$ and $D L V$ on $O P A$ is insignificant in this particular scenario.

As $P T V$ increases a similar phenomenon is observed. In this case it can also be observed that at a higher $P T V$, the system tries to achieve the maximum $O P A$ at a lower value of $R L$, other conditions remaining unchanged.

Table 1: $O P A$ vs. $R L$ at Varying Environmental Factors

\begin{tabular}{|c|c|c|c|c|c|c|c|c|}
\hline \multirow{2}{*}{\begin{tabular}{|l|} 
Fac- \\
tors
\end{tabular}} & \multirow{2}{*}{$\begin{array}{l}\text { Factor } \\
\text { Levels }\end{array}$} & \multicolumn{7}{|c|}{$R L$ (Hours) } \\
\hline & & 14 & 18 & 22 & 26 & 30 & 34 & 38 \\
\hline \multirow{3}{*}{$D L$} & 0.75 & 91.90 & 95.49 & 97.43 & 98.41 & 98.94 & 99.82 & 98.89 \\
\hline & 0.85 & 86.68 & 90.53 & 93.16 & 94.45 & 94.81 & 92.76 & 90.04 \\
\hline & 0.95 & 81.21 & 84.98 & 87.46 & 88.39 & 87.28 & 83.26 & 78.41 \\
\hline \multirow{3}{*}{$D L V$} & 0.10 & 86.68 & 90.53 & 93.16 & 94.45 & 94.81 & 92.76 & 90.04 \\
\hline & 0.55 & 85.72 & 89.68 & 92.34 & 93.66 & 94.00 & 92.02 & 89.08 \\
\hline & 1.00 & 83.45 & 87.55 & 90.38 & 91.77 & 92.26 & 90.16 & 86.99 \\
\hline \multirow{2}{*}{$P T V$} & 0.10 & 86.68 & 90.53 & 93.16 & 94.45 & 94.81 & 92.76 & 90.04 \\
\hline & 0.30 & 85.24 & 89.15 & 91.77 & 93.02 & 92.87 & 90.96 & 88.13 \\
\hline \multirow{3}{*}{$P U O$} & 0.05 & 86.68 & 90.53 & 93.16 & 94.45 & 94.81 & 92.76 & 90.04 \\
\hline & 0.15 & 87.07 & 90.80 & 92.89 & 93.70 & 93.90 & 92.61 & 91.17 \\
\hline & 0.25 & 87.47 & 90.91 & 92.54 & 92.99 & 93.09 & 92.17 & 91.87 \\
\hline \multirow{2}{*}{$D D T$} & Loose & 86.68 & 90.53 & 93.16 & 94.45 & 94.81 & 92.76 & 90.04 \\
\hline & Tight & 84.54 & 86.05 & 84.48 & 81.90 & 76.39 & 61.10 & 19.93 \\
\hline
\end{tabular}

Regarding the effect of $R L$ on $O P A$ under varying $P U O, O P A$ increases with $P U O$, at a lower $R L$, but when $R L$ is high a lower $P U O$ will attain a higher $O P A$.

At the "Tight" level of $D D T, O P A$ drops at a much faster rate with increasing $R L$ and also the system tries to attain the optimum $O P A$ at a lower value of $R L$. At the "Tight" level of $D D T$, the flow allowance of an order is smaller and also the tardiness cost penalty factor (i.e. Ktr) is higher. So to avoid a high tardiness penalty, the system rejects more orders to attain the maximum $O P A$, which is achievable at that condition.

\section{OPTIMAL CONTROL POLICY FOR GIVEN ENVIRONMENTAL CONDITIONS}

This section reports on an important and major aspect of this research which is to study how the system can be optimally controlled under different environments by adjusting the control parameters of the order acceptance rule. Also it is of interest to study how sensitive this choice of control parameters is with respect to variation in the environmental factors. The following sections study this for two classes of orders. The general approach to find out the optimal value of the control parameters $(R L$ and $H L)$ for a given environmental condition is detailed in the section below. These optimal values of $R L$ and $H L$ are plotted for dif- 
ferent environmental factors, in a range of figures in the following sections.

\subsection{General Approach to Find the Optimum}

To find out what should be the optimal control policy i.e. what should be the value of $R L$ and $H L$, so that the system performs the best, the approach taken is described below. This approach has two main steps:

(a) In the first step, a regression model is built which relates $O P A, R L$ and $H L$ and environmental factors viz. $D L, D L V, P T V, P U O$ and $D D T$.

(b) After the regression model is built, $O P A$ is optimized using this regression model with respect to $R L$ and $H L$ when other factors are set at specific values. The optimization is done by implementing the quasi-Newton search algorithm to find the direction of search while forward differencing is used to estimate the partial derivatives of the objective function. An initial estimate of the basic variables in one-dimensional search is done by quadratic extrapolation. Optimization of this type can be carried out using the Microsoft Excel solver.

To build a regression model, a cubic polynomial in seven factors is fitted to the observed values of $O P A$, which are obtained from a specified set of experiments. This set of experiments is determined by a D-optimal design, from a super set of full factorial design, under the condition that the effects appearing in the regression model are estimable. Each of these experiments in the chosen subset involved 5 replications, each of length 83520 time units. The statistics were cleared after a warm-up period of 11520 time units. These values were chosen so as to yield a confidence interval on $O P A$ whose half-width was less than $0.1 \%$ of the point estimate for $O P A$. This D-optimal design was carried out using the SAS statistical software package. See John and Draper (1975) for a review on D-optimality for regression designs.

\subsection{Influence of DL When Other Factors Are Fixed at Their Base Levels}

Figure 2 shows how the optimal control limits vary with $D L$ from 0.75 to 0.95 at a step of 0.05 keeping other factors at their base levels. It can be observed that (i) at $D L=0.75$, $R L$ is higher than $H L$, (ii) at a higher $D L$ the system chooses $H L$ higher than $R L$ to operate optimally, and (iii) in this higher range of $D L, H L$ does not vary much with respect to $D L$, while $R L$ decreases as $D L$ increases. Possible justifications for these three observed phenomena are as follows.

(i) When the system chooses $H L$ higher than $R L$ for optimal operation, it in effect reserves some space for the anticipated future urgent orders by rejecting some regular orders. In this scenario, only $5 \%$ of arriving orders are urgent which makes the arrival of urgent

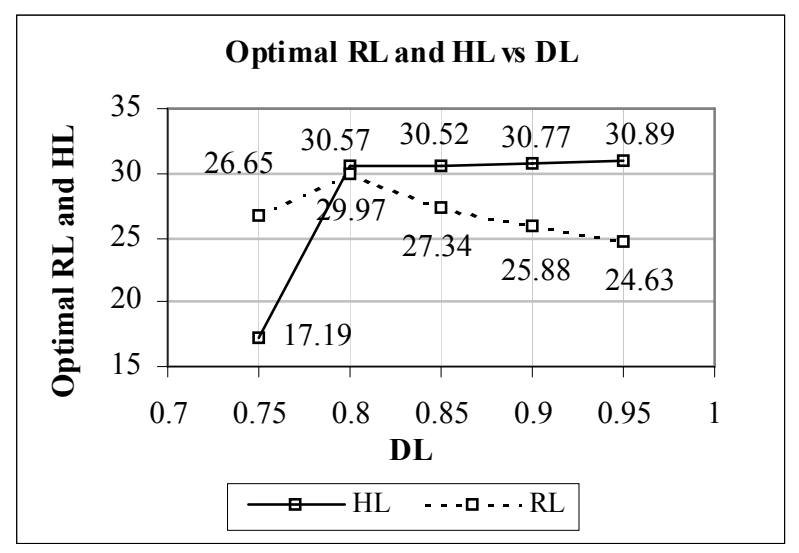

Figure 2: Optimum $R L$ and $H L$ vs. $D L$

orders relatively infrequent. So at a low $D L$ of 0.75 , reserving space for the urgent orders and thus rejecting the regular orders causes a rejection loss which is more than the extra revenue that could have been earned by accepting more urgent orders. So at $D L=$ 0.75 , to operate optimally, more regular orders are accepted by keeping $R L$ greater than $H L$.

(ii) At $D L$ greater than 0.75 however, the system shows a preference for the urgent orders over the regular orders. Here the system finds it beneficial to reserve some extra space for the urgent orders instead by rejecting some regular orders. The extra loss of revenue due to the rejection of regular orders (compared to the situation when $H L=R L$ ) is lower than the extra revenue earned by accepting more urgent orders. This acceptance of extra urgent orders would not have been possible, without excessively large increases in tardiness costs, if some extra regular orders were not rejected. However this does not necessarily mean that all urgent orders are accepted. Accepting all urgent orders might increase the loss due to tardiness of both classes of order. Due to the variability in the arrival process in the system there is an uneven frequency of arrival of urgent orders. If all the urgent orders are accepted by further lowering $R L$ (and hence by rejecting more regular orders), during any period of low frequency of arrival of urgent orders, the loss suffered by the system due to the rejection of regular orders cannot be made up by the revenue earned even by all the urgent orders in this period. So the total loss will increase through the increased rejection loss. On the other hand if $R L$ is not lowered further and all the urgent orders are accepted, this will lead to an increase in tardiness loss. So the optimum arrangement has been to reject a requisite amount of urgent and regular orders so as to 
minimize the sum total of the rejection and tardiness loss of both urgent and regular orders.

(iii) The system under consideration is a fixed capacity system and is working here under varying $D L$. To operate in an optimum fashion (i.e. producing the maximum OPA at a given situation), $R L$ and $H L$ must adjust to protect the system appropriately from the dynamics of the environment. In Figure 3, plots of $O P R L$ and $O P T L$ reveal that during the interval $D L=$



Figure 3: $O P R L$ and $O P T L$ vs. $D L$

0.75 and 0.80 , the system operates in an optimal fashion by accepting more orders while beyond that region, it relies on rejecting more orders. In this figure the values of $O P R L$ and $O P T L$ at a particular $D L$ are plotted when the system operates optimally. So beyond $D L=0.80$, the system treats orders of the two classes significantly differently. Rather it is apportioning the urgent and regular loads judiciously (through proper setting of $H L$ and $R L$ ) to maximize $O P A$. The system will always try to accept urgent orders as much as possible (under the constraint that the total loss is minimized, as explained in the context of observation (ii)). Figure 4 shows the plot of $U P R L$ and $R P R L$ with the values corresponding to the optimal operation of the system. As $D L$ is increased the fre-

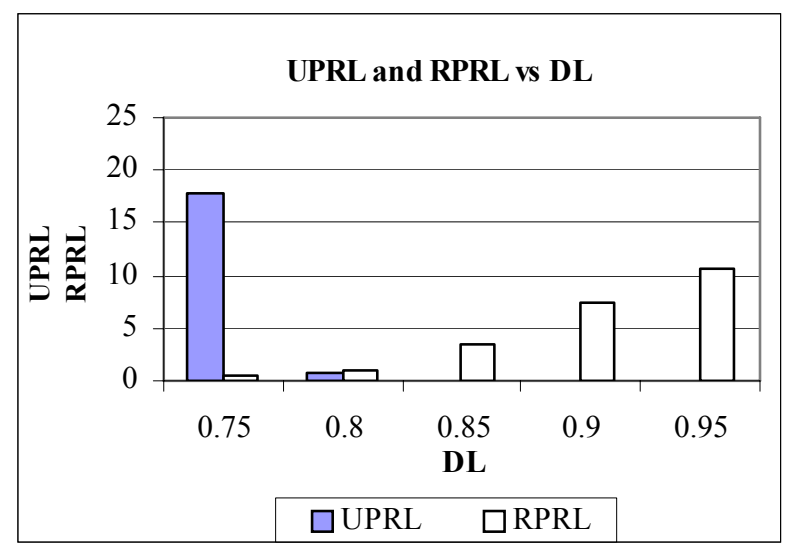

Figure 4: $U P R L$ and $R P R L$ vs. $D L$ quency of urgent orders will also increase and for $D L$ $>=0.85$, the system will try to accept these extra urgent orders fully, making UPRL to be zero. However, on an overall basis the system will operate optimally still by rejecting orders (leading to an increase in $O P R L)$ which is achieved through rejecting only regular orders. So for $D L>=0.85, R L$ will be reduced in order to be able to accept extra urgent orders without causing overly large tardiness penalties.

\subsection{Influence of $D L V$ When Other Factors Are Fixed at Their Base Levels}

Figure 5 suggests that there is little influence of $D L V$ on the choice of the optimal control limits when other factors are fixed at their base levels.

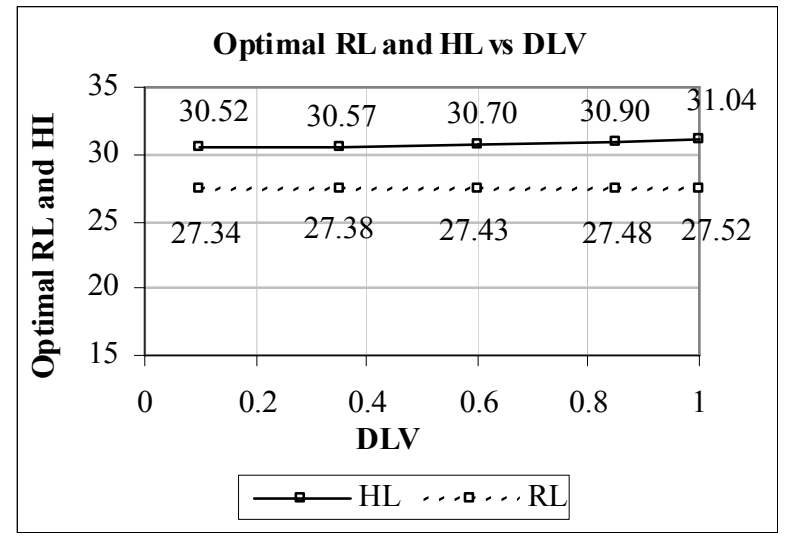

Figure 5: Optimal $R L$ and $H L$ vs. $D L V$

\subsection{Influence of $P T V$ When Other Factors Are Fixed at Their Base Levels}

Figure 6 is created by varying $P T V$ through $0.1,0.2,0.3$ with other factors at base levels. It shows that $P T V$ has little influence on the choice of the optimal control limits.

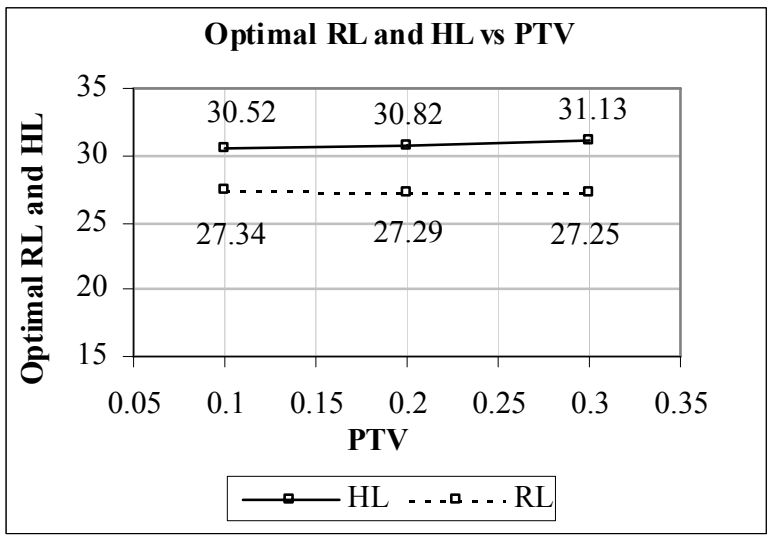

Figure 6: Optimum $R L$ and $H L$ vs. $P T V$ 


\subsection{Influence of PUO When Other Factors Are Fixed at Their Base Levels}

Figure 7 shows how the optimal choice of the control limits is influenced by changing $P U O$ from $5 \%$ to $25 \%$ at a step of $5 \%$ when other factors are fixed at their base levels. From the figure it can be observed that $H L$ remains higher than $R L$ over the whole range of $P U O$, and also that as $P U O$ increases, $H L$ remains relatively constant while $R L$

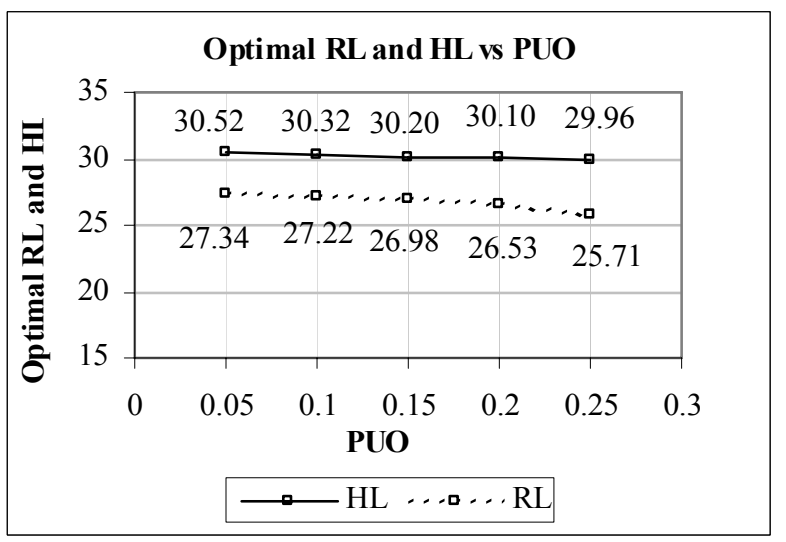

Figure 7: Optimal $R L$ and $H L$ vs. $P U O$

decreases slowly. To gain insight into this scenario, it is useful to look how $O P A, O P R L, O P T L, R P R L, U P T L$ and $R P T L$ vary with respect to $P U O$ when the system is operating in an optimal fashion. These data are shown in Table 2 in relation to the Section 5. The system finds it economic to keep room for the urgent orders and to do this a necessary amount of regular orders are rejected. Thus $H L$ remains higher than $R L$. At $D L=85 \%$, the system operates optimally by rejecting orders on an overall basis. If $P U O$ is increased, the system accepts all the extra urgent orders and $R L$ is lowered accordingly to reject the necessary quantity of regular orders so that the tardy loss does not become excessive.

\subsection{Influence of $D D T$ When Other Factors Are Fixed at Their Base Levels}

Figure 8 shows how the optimal choice of the control limits is influenced by varying $D D T$ across two different levels viz. "Loose" and "Tight" with other factors kept at their base levels. It can be observed that at the tight level, $R L$ reduces while $H L$ increases compared to the "Loose" level. This is expected because otherwise in the "Tight" level, the tardy loss will increase. So more orders are rejected. In both cases, $H L$ remains higher than $R L$. When the $D D T$ level changes from "Loose" to "Tight", the corresponding $O P A$ drops from 95.31 to 90.82 as seen in Table 2 .



Figure 8: Optimal $R L$ and $H L$ vs. $D D T$

\section{MAIN PERFORMANCE MEASURES UNDER OPTIMAL CONTROL}

This section reports on the behavior of the main performance measures of the manufacturing system when the system is controlled optimally under various environmental conditions and when urgent orders are allowed. Each row of Table 2 shows the values of these performance measures when the system operates with one environmental factor fixed at a level shown, while others are fixed at their respective reference levels and $R L$ and $H L$ at the values as shown. These values of $R L$ and $H L$ are the same values as obtained in the previous Section 4, which yields the maximum achievable $O P A$ at the environmental condition chosen. The values of different performance measures in each row of Table 2 were obtained from an appropriate simulation run that involved five replications, each of length 83,520 hours. The statistics were cleared after a warm-up period of 11,520 hours. These values were chosen to yield a confidence interval on $O P A$ with a half-width that was less than $0.1 \%$ of the point estimate for $O P A$.

Results show that as $D L$ increases $O P A$ drops significantly. At low $D L, O P R L$ decreases up to $D L=0.80$, after which it increases significantly, showing that the system maintains optimal performance by increasing the proportion of orders rejected. OPTL keeps low compared to $O P R L$ all across $D L>=0.85$. However when $D L V, P T V$ or $P U O$ increases, $O P A$ drops very little compared to when $D L$ changes. With increasing $D L, U P A$ and $R P A$ are also appropriately adjusted.

\section{CONCLUSIONS}

The order acceptance rule used in this research is a capacity-based rule and is parameterized. The rule can control the manufacturing system by adjusting the controllable parameters of the rule. Although the influence of similar capacity-based order acceptance rules on the performance of a manufacturing system was studied before, how to opti- 
Nandi and Rogers

Table 2: Performance Measures Under Optimal Control

\begin{tabular}{|c|c|c|c|c|c|c|c|c|c|c|c|}
\hline & & & 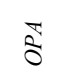 & $\S$ & 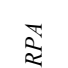 & $\frac{\vec{\jmath}}{\delta}$ & $\frac{\grave{z}}{\vdots}$ & $\stackrel{\gtrsim}{\grave{z}}$ & $\underset{\Omega}{a}$ & $\begin{array}{l}\hat{N} \\
\mathbb{S}\end{array}$ & $\underset{\&}{\mathbb{Z}}$ \\
\hline$\overline{D L}$ & $R L$ & $H L$ & & & & & & & & & \\
\hline 0.75 & 26.65 & 17.79 & 98.37 & 82.22 & 99.43 & $\begin{array}{l}1.47 \\
\end{array}$ & 17.72 & 0.40 & 0.16 & 0.06 & 0.16 \\
\hline$\overline{0.80}$ & 29.97 & 30.57 & 97.87 & 97.70 & 97.88 & 0.91 & 0.83 & 0.92 & 1.22 & 1.47 & 1.20 \\
\hline 0.85 & 27.34 & 30.52 & 95.31 & 97.56 & 95.16 & 3.29 & 0.02 & 3.51 & 1.40 & 2.42 & 1.33 \\
\hline 0.90 & 25.88 & 30.77 & 92.06 & 98.05 & 91.66 & 7.06 & 0.00 & 7.53 & 0.88 & 1.95 & 0.81 \\
\hline 0.95 & 24.63 & 30.89 & 88.65 & 97.14 & 88.09 & 10.06 & 0.00 & 10.72 & 1.30 & 2.86 & 1.19 \\
\hline
\end{tabular}

\begin{tabular}{|c|c|c|c|c|c|c|c|c|c|c|c|}
\hline$\overline{D L V}$ & $R L$ & $H L$ & & & & & & & & & \\
\hline 0.10 & 27.34 & 30.52 & 95.31 & 97.56 & 95.16 & 3.29 & 0.02 & 3.51 & 1.40 & 2.42 & 1.33 \\
\hline 0.35 & 27.38 & 30.57 & 95.09 & 97.57 & 94.93 & 3.51 & 0.05 & 3.73 & 1.40 & 2.38 & 1.34 \\
\hline 0.60 & 27.43 & 30.70 & 94.41 & 97.53 & 94.21 & 4.11 & 0.00 & 4.38 & 1.48 & 2.47 & 1.42 \\
\hline 0.85 & 27.48 & 30.90 & 93.51 & 97.26 & 93.26 & 4.89 & 0.10 & 5.21 & 1.60 & 2.64 & 1.53 \\
\hline 1.00 & 27.52 & 31.04 & 92.95 & 97.17 & 92.67 & 5.37 & 0.09 & 5.72 & 1.68 & 2.74 & 1.61 \\
\hline
\end{tabular}

\begin{tabular}{|c|c|c|c|c|c|c|c|c|c|c|c|}
\hline$P T V$ & $R L$ & $H L$ & & & & & & & & & \\
\hline 0.10 & 27.34 & 30.52 & 95.31 & 97.56 & 95.16 & 3.29 & 0.02 & 3.51 & 1.40 & 2.42 & 1.33 \\
\hline 0.20 & 27.29 & 30.82 & 94.79 & 97.17 & 94.63 & 3.53 & 0.07 & 3.76 & 1.68 & 2.76 & 1.61 \\
\hline 0.30 & 27.25 & 31.13 & 93.67 & 96.50 & 93.48 & 4.11 & 0.05 & 4.38 & 2.22 & 3.45 & 2.14 \\
\hline
\end{tabular}

\begin{tabular}{|c|c|c|c|c|c|c|c|c|c|c|c|}
\hline$P U O$ & $R L$ & $H L$ & & & & & & & & & \\
\hline 0.05 & 27.34 & 30.52 & 95.31 & 997.56 & 95.16 & 3.29 & 0.02 & 3.51 & 1.40 & 2.42 & 1.33 \\
\hline 0.10 & 27.22 & 30.32 & 95.09 & 97.28 & 94.79 & 3.16 & 0.04 & 3.60 & 1.74 & 2.69 & 1.61 \\
\hline 0.15 & 26.98 & 30.20 & 94.64 & 96.85 & 94.15 & 3.14 & 0.09 & 3.80 & 2.23 & 3.05 & 2.04 \\
\hline 0.20 & 26.53 & 30.10 & 94.17 & 96.39 & 93.49 & 3.11 & 0.15 & 4.02 & 2.72 & 3.46 & 2.50 \\
\hline 0.25 & 25.71 & 29.96 & 93.84 & 97.28 & 92.41 & 4.24 & 0.01 & 6.00 & 1.92 & 2.71 & 1.59 \\
\hline
\end{tabular}

\begin{tabular}{|c|c|c|c|c|c|c|c|c|c|c|c|}
\hline$D D T$ & $R L$ & $H L$ & & & & & & & & & \\
\hline Loose & 27.34 & 30.52 & 95.31 & 97.56 & 95.16 & 3.29 & 0.02 & 3.51 & 1.40 & 2.42 & 1.33 \\
\hline Tight & 18.22 & 38.33 & 90.82 & 99.84 & 90.23 & 9.15 & 0.00 & 9.75 & 0.03 & 0.16 & 0.02 \\
\hline
\end{tabular}

mally control a manufacturing system using this rule, given a set of environmental conditions, was not investigated. While using this rule in the scenario of two categories of order, interesting insights are gained on how the system judiciously apportions its earning of revenue from urgent and regular orders to adjust to the changing environmental conditions. The importance of this research lies in exploring the possibility to control a manufacturing system by such a rule. Exact implementation to a real system will necessitate customization of this rule in terms of reevaluating the effect of this rule on the specific system. However the possibility to optimally control the system will still hold good, as demonstrated in this paper. Real-life implementation of this rule will require infrastructures such as monitoring system, data collection, and modeling of the specific system among many others.

This research can be further extended along various avenues:

1. The implementation and analysis of such capacity-based rules should be extended to more complex manufacturing systems such as systems with assembled products and systems with distributed controls. To gain insight into how the rule manages the system with multi-class (more than two classes) orders will also be worth investigating.

2. This rule should be compared with other good order acceptance rules and possibly manufacturing control systems should be developed where the system can choose from a range of such order acceptance rules to work with depending on the environmental condition encountered and also the system can potentially switch to a different rule to its advantage, if necessary.

3. The optimum seeking methodology implemented here should be improved and for practical implementation, a reasonably fast and accurate optimum seeking method would be necessary. Also the effect of incorrect choice of the optimal values of the control parameters on the performance of the manufacturing system should be studied in more detail. 


\section{ACKNOWLEDGMENTS}

Thanks are due to the Natural Sciences and Engineering Research Council (NSERC) of Canada for partially funding some of the research reported in this paper.

\section{REFERENCES}

Bergamaschi, D., R. Cigolini, M. Perona, and A. Portoli. 1997. Order review and release strategies in a job shop environment: a review and a classification. International Journal of Production Research 35(2): 399-420.

Ebben, M.J.R., E.W. Hans, F.M.O. Weghuis. 2005. Workload based order acceptance in job shop environment. OR Spectrum 27: 107-122.

Ivanescu, C.V., Fransoo, J.C., Bertrand, J.W.M. 2002. Makespan estimation and order acceptance in batch process industries when processing times are uncertain. OR Spectrum 24(4): 467-495.

John, R.C. St. and N.R. Draper. 1975, D-optimality for regression designs: a review. Technometrics 17(1): 1523.

Lippman, S.A. 1975. Applying a new device in the optimization of exponential queuing systems. Operations Research 23: 687-710.

Lippman, S.A. and S. Ross. 1971. The streetwalker's dilemma: a job shop model. SIAM Journal of Applied Mathematics 20: 336-344.

Miller, B.I. 1969. A queuing reward system with several customer classes. Management Sciences 16: 234-245.

Nandi, A. and P. Rogers. 2004. Using simulation to make order acceptance/rejection decisions. Simulation: Transactions of the Society for Modeling and Simulation International 80: 131-142.

Nandi, A. and P. Rogers. 2003. Behavior of an order release mechanism in a make-to-order manufacturing system with selected order acceptance. In Proceedings of the 2003 Winter Simulation Conference,eds. S. Chick, P.J. Sanchez, D. Ferrin, and D.J. Morris. 12511259. Piscataway, New Jersey: Institute of Electrical and Electronics Engineers.

Philipoom, P.R. and T.D. Fry. 1992. Capacity-based order review/release strategies to improve manufacturing performance. International Journal of Production Research 30(11): 2559-2572.

Scott, M. 1970. Queuing with control on the arrival of certain types of customers. CORS Journal 8: 75-86.

Scott, M. 1969. A queuing process with some discrimination. Management Sciences 16: 227-233.

ten Kate, H.A. 1994. Towards a better understanding of order acceptance. International Journal of Production Economics 37: 139-152.

Wester, F.A.W., J. Wijngaard, and W.H.M. Zijm. 1992. Order acceptance strategies in a production-to-order environments with setup times and due dates. International Journal of Production Research 30(6): 13131326.

Wight, O. 1970. Input/output control a real handle on lead time. Production and Inventory Management 11(3): 931.

\section{AUTHOR BIOGRAPHIES}

AMITAVA NANDI is a Research and Technology Advisor in the SR\&ED division of Canada Revenue Agency in Ottawa, Canada. His interests include system design and analysis, productivity improvement, manufacturing systems modeling, statistical process control, and multivariate data analysis. He holds a Ph.D. from the University of Calgary (in Canada), and an M.Sc. from Brunel University (in England). $\mathrm{He}$ can be contacted by email at <amitava_nandi@rediffmail.com>.

PAUL ROGERS is an Associate Professor in the Department of Mechanical and Manufacturing Engineering at the University of Calgary. His research and teaching interests include production planning and control systems, manufacturing strategy, distributed and dynamic scheduling in electronics manufacturing, object-oriented modeling for intelligent manufacturing, simulation of intelligent agent systems, and models for the design and analysis of manufacturing systems. He is a Professional Engineer registered with the Association of Professional Engineers, Geologists, and Geophysicists of Alberta (APEGGA), a member of IIE, $A P I C S$, and INFORMS, and serves on the Editorial Board of the International Journal of Computer Integrated Manufacturing. He holds Ph.D. and M.Eng. degrees from Cambridge University in England. He can be contacted by email at $<$ rogers@ucalgary . ca $>$. 